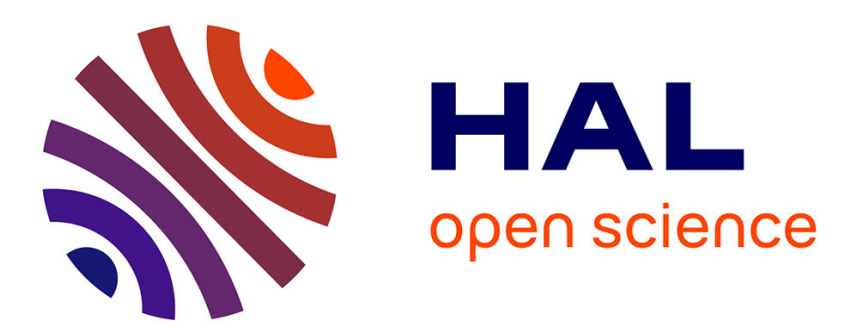

\title{
Stability analysis and stabilisation of switched nonlinear systems
}

\author{
Carlos Alberto Cavichioli Gonzaga, Marc Jungers, Jamal Daafouz
}

\section{To cite this version:}

Carlos Alberto Cavichioli Gonzaga, Marc Jungers, Jamal Daafouz. Stability analysis and stabilisation of switched nonlinear systems. International Journal of Control, 2012, 85 (7), pp.822-829. 10.1080/00207179.2012.666855 . hal-00696795

\section{HAL Id: hal-00696795 \\ https://hal.science/hal-00696795}

Submitted on 15 Jul 2013

HAL is a multi-disciplinary open access archive for the deposit and dissemination of scientific research documents, whether they are published or not. The documents may come from teaching and research institutions in France or abroad, or from public or private research centers.
L'archive ouverte pluridisciplinaire HAL, est destinée au dépôt et à la diffusion de documents scientifiques de niveau recherche, publiés ou non, émanant des établissements d'enseignement et de recherche français ou étrangers, des laboratoires publics ou privés. 


\title{
Regular Paper
}

\section{Stability analysis and stabilization of switched nonlinear systems: a switched nonlinearity-dependent Lyapunov function}

\author{
Carlos A. C. Gonzaga ${ }^{a}$ and Marc Jungers ${ }^{a *}$ and Jamal Daafouz ${ }^{a, b}$ \\ ${ }^{a}$ Centre de Recherche en Automatique de Nancy, Nancy-Université, CNRS, 2 avenue de la Forêt de \\ Haye F-54516 Vandoeuvre-les-Nancy, France; ${ }^{2}$ Institut Universitaire de France \\ (v3.5 released August 2008)
}

\begin{abstract}
A class of switched non-quadratic Lyapunov functions is considered in this paper. The function is associated with discrete-time switched systems subject to mode-dependent cone bounded nonlinearities and saturation actuator. These Lyapunov functions depend on the switched nonlinearities and on the active mode, instead of the time-invariant Lur'e-type function. The only assumption considered here is the mode-dependent sector condition, without constraint related to the slope of the nonlinearities. The stability analysis and control design problems lead to linear matrix inequalities (LMI) conditions. Optimization problems are provided to cope with both issues in order to maximize the size of the basin of attraction estimate, which may be composed of disconnected sets. Numerical examples illustrate via comparisons that we improve the results of the literature.
\end{abstract}

Keywords: Lyapunov function; cone bounded nonlinearity; local stability and stabilization; input saturation; basin of attraction estimate; LMIs.

\section{Introduction}

Switched systems are a subclass of hybrid systems characterized by a number of subsystems and a switching strategy imposing the active mode at each time (Liberzon 2003). In a large number of contributions, the switching modes are formulated as linear ones. This is, in general, only an approximation for physical systems models to be valid in a limited domain. Nonetheless, because of the actuators cannot provide unbounded magnitude signals, the resulting dynamics could be nonlinear. Thus, it is reasonable to consider some nonlinearities dependent on the state and the control input. These nonlinearities are naturally mode-dependent and should be taken into account to refine the modelling step.

The saturation nonlinearity draw attention in the classical literature of control design. Two representations of the saturation are mostly considered (Tarbouriech et al. 2011): as a polytope and as a cone bounded nonlinearity. These models were also used to study switched systems subject to saturation, (Benzaouia et al. 2004, Lu and Lin 2008, Jungers et al. 2009, 2010). References (Jungers et al. 2009, 2010) deal with more general systems including, in addition, mode-dependent cone-bounded nonlinearities, as investigated in this paper.

Stability of linear systems connected to a cone bounded nonlinearity has been widely studied, so-called Lur'e problem (Khalil 2002). In continuous-time, a dedicated Lyapunov function has been proposed, named Lur'e-type function, which is composed of a quadratic term with respect to the state and an integral term of the nonlinearity. The integral is justified because it induces the presence of the nonlinearity in the Lyapunov time-derivative. Stability conditions are, then, achieved only by managing with the sector condition. In discrete-time, the idea of using the

*Corresponding author. Email: marc.jungers@cran.uhp-nancy.fr 
same function has also been considered (Haddad and Bernstein 1994, Kapila and Haddad 1996, Gomes da Silva Jr. et al. 2001, Jury and Lee 1964a,b, Sharma and Singh 1981). Nevertheless, additional assumptions about the nonlinearity slope are required to upper-bound the remaining integral term in the Lyapunov difference. At last but not least, the Lur'e function requires timeinvariance of the nonlinearity. Extending such a tool to switched systems with mode-dependent nonlinearities is difficult due to the time dependency.

In this paper, we tackle the problems of stability analysis and stabilization of discrete-time switched systems including switched nonlinearities and input saturation. The same issue has been investigated in (Gonzaga et al. 2011) in the simple case where the systems do not switch. The main contribution here is to consider a switched Lyapunov function which depends on the switched cone bounded nonlinearities without additional assumptions on their slope. Sufficient conditions for stability analysis are provided as linear matrix inequalities (LMI). On the other hand, the stabilization purpose is directly given by bilinear matrix inequalities (BMI) which are relaxed through the Finsler's Lemma obtaining sufficient conditions as LMIs.

The paper is organized as follows: Section 2 is devoted to the system description, some preliminaries and a switched nonlinearity-dependent Lyapunov function is presented. The main results related to the stability analysis problem are provided in Section 3 and a numerical example is given. Section 4 is dedicated to the stabilization issue and the same example of Section 3 is considered. Concluding remarks are given in Section 5.

Notation: Components of vector $x \in \mathbb{R}^{n}$ are denoted $x_{(\ell)}, \forall \ell=1, \ldots, n$. Vectors inequalities are component-wise: $x \geq 0$ means $x_{(\ell)} \geq 0$ and $x \geq y$ means $x_{(\ell)}-y_{(\ell)} \geq 0 . A_{(\ell)}\left(\right.$ resp. $\left.A_{i,(\ell)}\right)$ is the $\ell$-th row of matrix $A$ (resp. $A_{i}$ ). For two symmetric matrices $A, B, A>B$ means that $A-B$ is positive definite. $A^{\prime}$ is the transpose of $A . I_{n}$ (resp. $0_{n}$ ) and $0_{m \times n}$ are the $n$-order identity (null) matrix and the $m \times n$-order null matrix, respectively. Operator $\operatorname{diag}[x]$ means a diagonal matrix obtained from vector $x$. Symbol $\star$ stands for symmetric block in matrices. The ellipsoidal set $\mathcal{E}(M, \gamma)$ associated with $M>0$ is given by $\left\{x \in \mathbb{R}^{n} ; x^{\prime} M x \leq \gamma\right\}$ and the shortcut $\mathcal{E}(M)=\mathcal{E}(M, 1)$ is used. For a given matrix $X \in \mathbb{R}^{n \times n}, \mathbf{H e}(X)=X+X^{\prime}$.

\section{$2 \quad$ System description and preliminaries}

Consider the discrete-time switching system composed of $N$ nonlinear modes $\left(N \in \mathbb{N}^{*}\right)$

$$
\begin{aligned}
x_{k+1} & =A_{\sigma(k)} x_{k}+F_{\sigma(k)} \varphi_{\sigma(k)}\left(y_{k}\right)+B_{\sigma(k)} \operatorname{sat}\left(u_{k}\right), \\
y_{k} & =C_{\sigma(k)} x_{k},
\end{aligned}
$$

where $x_{k} \in \mathbb{R}^{n}, u_{k} \in \mathbb{R}^{m}, y_{k} \in \mathbb{R}^{p}$ and $\varphi_{\sigma(k)}(\cdot) \in \mathbb{R}^{p}$ are respectively the state, input, output and the active nonlinearity vector of the system (1)-(2) at the time instant $k \in \mathbb{N}$. This system will satisfy the following assumptions.

Assumption 2.1 The switching rule $\sigma: \mathbb{N} \rightarrow \mathbb{N}^{*}$ takes its values in the finite set $\mathcal{I}_{N}=\{1, \cdots, N\}$ and is assumed to be not known a priori, but its current value $\sigma(k)$ to be available in real-time.

The notation $M_{\sigma(k)}$ means that at each time $k, M_{\sigma(k)}$ takes its values in the set $\left\{M_{1}, \cdots, M_{N}\right\}$ indexed by $\sigma(k)$. The matrices $A_{i}, B_{i}, C_{i}$ and $F_{i}, \forall i \in \mathcal{I}_{N}$, have appropriate dimensions.

Assumption 2.2 The $N$ nonlinearities $\varphi_{i}(\cdot): \mathbb{R}^{p} \rightarrow \mathbb{R}^{p}$ associated with each mode $i \in \mathcal{I}_{N}$ are assumed to be decentralized and to satisfy their own cone bounded sector condition (Khalil 2002). These sector conditions will be mode-dependent.

This assumption, which writes $\varphi_{i}(\cdot) \in\left[0_{p}, \Omega_{i}\right]$ with a conventional abuse of notations (Khalil 2002), means that $\varphi_{i}(0)=0$ and there exist $N$ diagonal positive definite matrices $\Omega_{i} \in \mathbb{R}^{p \times p}$ such that, $\forall y \in \mathbb{R}^{p}, \forall i \in \mathcal{I}_{N}, \varphi_{i,(\ell)}(y)\left[\varphi_{i}(y)-\Omega_{i} y\right]_{(\ell)} \leq 0, \forall \ell=1, \cdots, p$. Hence, we have the 
following inequality:

$$
\operatorname{SC}\left(\varphi_{i}(\cdot), y, \Lambda_{i}\right)=\varphi_{i}^{\prime}(y) \Lambda_{i}\left[\varphi_{i}(y)-\Omega_{i} y\right] \leq 0,
$$

where $\Lambda_{i} \triangleq \operatorname{diag}\left\{\lambda_{q, i}\right\}_{q=1 ; \cdots ; p} \in \mathbb{R}^{p \times p}$ are any positive definite diagonal matrices. Note that $\Omega_{i}$ is given by the designer and assumed to be known hereafter for each mode $i \in \mathcal{I}_{N}$.

It is simple to show that relation (3) is equivalent to $\left[\Omega_{i} y\right]_{(\ell)}^{\prime}\left[\varphi_{i}(y)-\Omega_{i} y\right]_{(\ell)} \leq 0, \forall \ell=1, \cdots, p$, $\forall y \in \mathbb{R}^{p} ; \forall i \in \mathcal{I}_{N}$, which implies, with $\Lambda_{i}$ diagonal positive definite, that

$$
0 \leq \varphi_{i}^{\prime}(y) \Lambda_{i} \varphi_{i}(y) \leq \varphi_{i}^{\prime}(y) \Lambda_{i} \Omega_{i} y \leq y^{\prime} \Omega_{i}^{\prime} \Lambda_{i} \Omega_{i} y, \forall y \in \mathbb{R}^{p} .
$$

Assumption 2.3 The control input $u_{k}$ is subject to actuation limits, and the standard saturation function is considered: $\operatorname{sat}\left(u_{k}\right)_{(\ell)}=\operatorname{sign}\left(\left(u_{k}\right)_{(\ell)}\right) \min \left(\rho_{(\ell)},\left|\left(u_{k}\right)_{(\ell)}\right|\right), \forall \ell=1, \ldots, m$. The vector $0_{m}<\rho \in \mathbb{R}^{m}$ is assumed to be given.

Throughout this paper, the following class of control law is considered (Jungers et al. 2009):

$$
u_{k}=K_{\sigma(k)} x_{k}+\Gamma_{\sigma(k)} \varphi_{\sigma(k)}\left(y_{k}\right)
$$

where the $m \times n$ matrix $K_{\sigma(k)}$ is a switching state feedback gain and the $m \times p$ matrix $\Gamma_{\sigma(k)}$ is a switching feedback gain associated with the active nonlinearity $\varphi_{\sigma(k)}(\cdot)$. If $\Gamma_{\sigma(k)} \neq 0_{m \times p}$, the feedback control law requires the following assumption

Assumption 2.4 The value of the active nonlinearity $\varphi_{\sigma(k)}\left(y_{k}\right)$ is assumed to be known as a signal (Arcak et al. 2003) at time $k$, either by model estimate or measuring.

The saturation is described as a dead-zone nonlinearity $\Psi\left(u_{k}\right)=u_{k}-\operatorname{sat}\left(u_{k}\right)$. By replacing $u_{k}$ defined in (5) and using $\Psi\left(u_{k}\right)$ into (1), the closed-loop model is given by

$$
x_{k+1}=A_{\mathrm{cl}, \sigma(k)} x_{k}+F_{\mathrm{cl}, \sigma(k)} \varphi_{\sigma(k)}\left(y_{k}\right)-B_{\sigma(k)} \Psi\left(u_{k}\right),
$$

where $A_{\mathrm{cl}, i}=A_{i}+B_{i} K_{i}$ and $F_{\mathrm{cl}, i}=F_{i}+B_{i} \Gamma_{i}, \forall i \in \mathcal{I}_{N}$.

The following set is used to characterize the dead-zone as belonging to a generalized sector condition. For given matrices $H_{i} \in \mathbb{R}^{m \times(n+p)}, i \in \mathcal{I}_{N}$, consider

$$
\mathcal{S}\left(\left\{H_{i}\right\}_{i \in \mathcal{I}_{N}}, \rho\right)=\left\{\theta \in \mathbb{R}^{n+p} ;-\rho \leq H_{i} \theta \leq \rho, \forall i \in \mathcal{I}_{N}\right\}
$$

Lemma 2.5 Let $m \times(n+p)$-matrices $\widehat{K}_{\sigma}=\left[\begin{array}{ll}K_{\sigma} & \Gamma_{\sigma}\end{array}\right]$ and $\widehat{J}_{\sigma}=\left[\begin{array}{lll}J_{1, \sigma} & J_{2, \sigma}\end{array}\right], \quad \sigma \in \mathcal{I}_{N}$. If the vector $\widehat{x}_{k}=\left[\begin{array}{ll}x_{k}^{\prime} & \varphi_{\sigma}^{\prime}\left(y_{k}\right)\end{array}\right]^{\prime}$ is an element of $\mathcal{S}\left(\left\{\widehat{K}_{\sigma}-\widehat{J}_{\sigma}\right\}_{\sigma \in \mathcal{I}_{N}}, \rho\right)$, the nonlinearity $\Psi\left(u_{k}\right)$ satisfies the following sector condition

$$
\mathrm{SC}_{u_{k}}=\Psi^{\prime}\left(u_{k}\right) U_{\sigma(k)}^{-1}\left[\Psi\left(u_{k}\right)-J_{1, \sigma(k)} x_{k}-J_{2, \sigma(k)} \varphi_{\sigma(k)}\left(y_{k}\right)\right] \leq 0, \quad \forall \sigma \in \mathcal{I}_{N}
$$

with $u_{k}$ defined in (5), for any diagonal positive definite matrix $U_{\sigma} \in \mathbb{R}^{m \times m}$.

Proof The proof follows the same lines of (Tarbouriech et al. 2006, Lemma 1).

After presenting the studied class of systems and the necessary background, the following problems will be addressed in this paper.

Problem 2.6 (Local Stability Analysis) Given the switched gains $K_{i}$ and $\Gamma_{i}\left(i \in \mathcal{I}_{N}\right)$ of the control law (5), and under Assumptions 2.1-2.4, determine a region in the state space, as large as possible, included in the basin of attraction $\mathcal{B}_{0}$ of the system (6), allowing to conclude the local asymptotic stability for any switching rule. 
Problem 2.7 (Closed-loop Stabilization) Determine the control law (5) such that the closed-loop system (6) is locally asymptotically stable for any switching rule, in a region as large as possible, included in the basin of attraction $\mathcal{B}_{0}$.

Instead of using a switched quadratic Lyapunov function, as considered in (Jungers et al. 2009), to study both problems, we want to investigate a function which takes the cone bounded nonlinearity $\varphi_{i}(\cdot)$ into account. However, the wide-spread Lur'e-type function (Khalil 2002) cannot be employed due to the mode-dependent cone bounded nonlinearities. By taking advantage of the class of Lyapunov functions presented in our recent work (Gonzaga et al. 2011), an extension to cover the time-varying nature of these nonlinearities is possible. Moreover it will allow to improve the results of the literature considering switched quadratic Lyapunov functions and to relaxe the bilinear nature of control design sufficient conditions.

\subsection{Switched nonlinearity-dependent Lyapunov functions}

The following class of non-quadratic switched Lyapunov candidate functions will be considered as main tool in this paper. Let:

$$
V:\left\{\begin{array}{l}
\mathcal{I}_{N} \times \mathbb{R}^{n} \times \mathbb{R}^{p} \longrightarrow \mathbb{R}, \\
\left(i, x, \varphi\left(C_{i} x\right)\right) \longmapsto x^{\prime} P_{i} x+2 \varphi^{\prime}\left(C_{i} x\right) \Delta_{i} \Omega_{i} C_{i} x,
\end{array}\right.
$$

where matrices $P_{i} \in \mathbb{R}^{n \times n}$ are symmetric positive definite and $\Delta_{i} \in \mathbb{R}^{p \times p}$ are diagonal positive semi-definite $\left(i \in \mathcal{I}_{N}\right)$. It is noteworthy that, by setting $\Delta_{i}=0_{p}\left(\forall i \in \mathcal{I}_{N}\right)$, the switched quadratic Lyapunov function is recovered and, thus, it may be considered as a particular case of (9).

From Inequalities (4), it is possible to define a lower and an upper bounds given by two switched quadratic functions

$$
x^{\prime} P_{i} x \leq V\left(i ; x ; \varphi_{i}\left(C_{i} x\right)\right) \leq x^{\prime}\left(P_{i}+2 C_{i}^{\prime} \Omega_{i}^{\prime} \Delta_{i} \Omega_{i} C_{i}\right) x, \quad \forall i \in \mathcal{I}_{N} .
$$

The function $V$ can be considered as a candidate, because it verifies the following properties:

- $V\left(i ; x ; \varphi_{i}\left(C_{i} x\right)\right) \geq 0, \forall x \in \mathbb{R}^{n}, \forall i \in \mathcal{I}_{N}$, due to the left inequality in (10);

- $V\left(i ; x ; \varphi_{i}\left(C_{i} x\right)\right)=0$, if and only if $x=0$, because $P_{i}>0_{n}, \forall i \in \mathcal{I}_{N}$;

- $V\left(i ; x ; \varphi_{i}\left(C_{i} x\right)\right)$ is unbounded, i.e., $V\left(i ; x ; \varphi_{i}\left(C_{i} x\right)\right) \rightarrow \infty$ whenever $\|x\| \rightarrow \infty, \forall i \in \mathcal{I}_{N}$.

The Lyapunov difference is defined as

$$
\delta_{k} V=V\left(\sigma(k+1) ; x_{k+1} ; \varphi_{\sigma(k+1)}\left(C_{\sigma(k+1)} x_{k+1}\right)\right)-V\left(\sigma(k) ; x_{k} ; \varphi_{\sigma(k)}\left(C_{\sigma(k)} x_{k}\right)\right) .
$$

The Level Set $L_{V}(\gamma)$ associated with $V\left(i ; x ; \varphi_{i}\left(C_{i} x\right)\right)$ and a $\gamma>0$ is given by

$$
\begin{aligned}
L_{V}(\gamma) & =\left\{x \in \mathbb{R}^{n} ; V\left(i ; x ; \varphi_{i}\left(C_{i} x\right)\right) \leq \gamma, \forall i \in \mathcal{I}_{N}\right\} \\
& =\bigcap_{i \in \mathcal{I}_{N}}\left\{x \in \mathbb{R}^{n} ; V\left(i ; x ; \varphi_{i}\left(C_{i} x\right)\right) \leq \gamma\right\},
\end{aligned}
$$

and is naturally related to the two ellipsoids intersections associated with the upper and lowerbounding switched quadratic functions

$$
\bigcap_{i \in \mathcal{I}_{N}} \mathcal{E}\left(P_{i}, \gamma\right) \supseteq L_{V}(\gamma) \supseteq \bigcap_{i \in \mathcal{I}_{N}} \mathcal{E}\left(P_{i}+2 C_{i}^{\prime} \Omega_{i}^{\prime} \Delta_{i} \Omega_{i} C_{i}, \gamma\right)
$$

Inclusion (13) emphasizes an important property of function $V\left(i ; x ; \varphi_{i}\left(C_{i} x\right)\right)$ : because of taking 
the nonlinearities $\varphi_{i}(\cdot)\left(i \in \mathcal{I}_{N}\right)$ into account, the set $L_{V}(\gamma)$ may be non-convex and disconnected. Hence, the level set $L_{V}(1)$ of our function $V\left(i ; x ; \varphi_{i}\left(C_{i} x\right)\right)$ will be considered to study Problems 2.6 and 2.7.

Remark 1: The non-convexity and the disconnection of the level set are important properties, justified by the fact that the transition between $x_{k}$ and $x_{k+1}$ in the discrete-time domain is not continuously done.

\section{Local Stability Analysis}

This section is dedicated to solve Problem 2.6. Consider the auxiliary matrices

$$
\begin{aligned}
\mathcal{M}_{0, i, j} & =\left[\begin{array}{ccccc}
P_{j} & 0_{n} & 0_{n \times p} & 0_{n \times m} & 0_{n \times p} \\
\star & -P_{i} & C_{i}^{\prime} \bar{\Omega}_{i}\left[T_{i}-\Delta_{i}\right] & J_{1, i}^{\prime} & 0_{n \times p} \\
\star & \star & -2 T_{i} & J_{2, i}^{\prime} & 0_{p} \\
\star & \star & \star & -2 U_{i} & 0_{m \times p} \\
\star & \star & \star & \star & 2 \Delta_{j}
\end{array}\right] ; \mathcal{M}_{1, i, j}=\left[\begin{array}{c}
I_{n} \\
-\left(A_{i}+B_{i} K_{i}\right)^{\prime} \\
-\left(F_{i}+B_{i} \Gamma_{i}\right)^{\prime} \\
U_{i}^{\prime} B_{i}^{\prime} \\
0_{p \times n}
\end{array}\right] ; \\
\mathcal{M}_{2, i, j}^{\prime} & =\left[\begin{array}{llll}
0_{p \times n}-\Omega_{j} C_{j}\left(A_{i}+B_{i} K_{i}\right) & \left.-\Omega_{j} C_{j}\left(F_{i}+B_{i} \Gamma_{i}\right) \Omega_{j} C_{j} B_{i} U_{i} I_{p}\right]
\end{array}\right.
\end{aligned}
$$

$\mathcal{N}_{G_{j}}=\left[-G_{j} ; 0_{n \times(m+2 p+n)}\right] ; \mathcal{N}_{\Theta_{j}}=\left[0_{p \times(m+2 n+p)} ;-\Theta_{j}\right]$, where $\Theta_{j}=W_{j}+\Delta_{j}, \forall j \in \mathcal{I}_{N}$, which will be used in the next theorem

Theorem 3.1 For given matrices $K_{i} \in \mathbb{R}^{m \times n}, \Gamma_{i} \in \mathbb{R}^{m \times p}$ and fixed positive diagonal matrices $U_{i} \in \mathbb{R}^{m \times m}\left(i \in \mathcal{I}_{N}\right)$, consider matrices $G_{i} \in \mathbb{R}^{n \times n}, J_{1, i} \in \mathbb{R}^{m \times n}, J_{2, i} \in \mathbb{R}^{m \times p}$, symmetric positive definite matrices $P_{i} \in \mathbb{R}^{n \times n}$ and positive semi-definite diagonal matrices $\Delta_{i} \in \mathbb{R}^{p \times p}$ and positive definite diagonal matrices $R_{i}, Q_{i}, T_{i}, \Theta_{i} \in \mathbb{R}^{p \times p}$, and a scalar $\mu$ in the optimization problem

$$
\begin{aligned}
& \min _{\mu, G_{i}, P_{i}, J_{1, i}, J_{2, i}, Q_{i}, R_{i}, T_{i}, \Theta_{i}, \Delta_{i}}^{\mu} \\
& \text { subject to LMIs: } \\
& {\left[\begin{array}{cc}
\mu I_{n}-P_{i}-C_{i}^{\prime} \bar{\Omega}_{i}\left[R_{i}+\Delta_{i}\right] \\
\star & 2 R_{i}
\end{array}\right]>0_{(n+p)}, \quad \forall i \in \mathcal{I}_{N},} \\
& {\left[\begin{array}{ccc}
P_{i} & C_{i}^{\prime} \bar{\Omega}_{i}\left[\Delta_{i}-Q_{i}\right] & \left(K_{i}-J_{1, i}\right)_{(\ell)}^{\prime} \\
\star & 2 Q_{i} & \left(\Gamma_{i}-J_{2, i}\right)_{(\ell)}^{\prime} \\
\star & \star & \rho_{(\ell)}^{2}
\end{array}\right]>0_{(n+p+1)}, \quad \forall i \in \mathcal{I}_{N} ; \forall \ell=1, \cdots, m ;} \\
& \mathcal{M}_{0, i, j}+\operatorname{He}\left(\mathcal{M}_{1, i, j} \mathcal{N}_{G_{i}}+\mathcal{M}_{2, i, j} \mathcal{N}_{\Theta_{j}}\right)<0_{(2 n+2 p+m)}, \forall(i, j) \in \mathcal{I}_{N}^{2} .
\end{aligned}
$$

Then, Problem 2.6 is solved with an estimate of $\mathcal{B}_{0}$ of system (6) given by the set $L_{V}(1)$, induced by the Lyapunov function (9).

Proof By noting the block matrices as defined in (14)-(15), Inequality (18) is the same as the 
following one

$$
\left[\begin{array}{ccccc}
P_{j}-G_{j}-G_{j}^{\prime} G_{j}^{\prime}\left(A_{i}+B_{i} K_{i}\right) & G_{j}^{\prime}\left(F_{i}+B_{i} \Gamma_{i}\right) & -G_{j}^{\prime} B_{i} U_{i} & 0_{n \times p} \\
\star & -P_{i} & C_{i}^{\prime} \bar{\Omega}_{i}\left[T_{i}-\Delta_{i}\right] & J_{1, i}^{\prime} & \left(A_{i}+B_{i} K_{i}\right)^{\prime} C_{j}^{\prime} \bar{\Omega}_{j} \Theta_{j} \\
\star & \star & -2 T_{i} & J_{2, i}^{\prime} & \left(F_{i}+B_{i} \Gamma_{i}\right)^{\prime} C_{j}^{\prime} \bar{\Omega}_{j} \Theta_{j} \\
\star & \star & \star & -2 U_{i} & -U_{i} B_{i}^{\prime} C_{j}^{\prime} \bar{\Omega}_{j} \Theta_{j} \\
\star & \star & \star & \star & -2\left[\Theta_{j}-\Delta_{j}\right]
\end{array}\right]<0_{(2 n+2 p+m)} .
$$

Hence, if Inequality (18) holds, one has $\Theta_{j}-\Delta_{j}>0_{p}$, which combined with $\Delta_{j} \geq 0_{p}$, implies $\Theta_{j}>$ $0_{p}$, and so $W_{j}>0_{p}\left(\forall j \in \mathcal{I}_{N}\right)$. In addition, it means $P_{j}-G_{j}^{\prime}-G_{j}<0_{n}$ and $P_{i}>0_{n}$. Thus, $G_{j}$ is of full rank and $-G_{j}^{\prime} P_{j}^{-1} G_{j} \leq P_{j}-G_{j}^{\prime}-G_{j}$ (see (Daafouz and Bernussou 2001)). This implies, combined with the change of basis $\operatorname{diag}\left[G_{j}^{-1} ; I_{n+p} ; U_{i}^{-1}, I_{p}\right]$, and a Schur complement (Boyd et al. 1994)

$$
\left[\begin{array}{c}
A_{\mathrm{cl}, i}^{\prime} \\
F_{\mathrm{cl}, i}^{\prime} \\
-B_{i}^{\prime} \\
0_{p \times n}
\end{array}\right] P_{j}\left[\begin{array}{c}
A_{\mathrm{cl}, i}^{\prime} \\
F_{\mathrm{cl}, i}^{\prime} \\
-B_{i}^{\prime} \\
0_{p \times n}
\end{array}\right]^{\prime}+\left[\begin{array}{cccc}
-P_{i} C_{i}^{\prime} \Omega_{i}\left[T_{i}-\Delta_{i}\right] & J_{1, i}^{\prime} U_{i}^{-1} & A_{\mathrm{cl}, i}^{\prime} C_{j}^{\prime} \Omega_{j}\left[W_{j}+\Delta_{j}\right] \\
\star & -2 T_{i} & J_{2, i}^{\prime} U_{i}^{-1} & F_{\mathrm{cl}, i}^{\prime} C_{j}^{\prime} \Omega_{j}\left[W_{j}+\Delta_{j}\right] \\
\star & \star & -2 U_{i}^{-1} & -B_{i}^{\prime} C_{j}^{\prime} \Omega_{j}\left[W_{j}+\Delta_{j}\right] \\
\star & \star & \star & -2 W_{j}
\end{array}\right]<0_{(2 n+2 p+m)}
$$

By multiplying Inequality (20) on the right by $\left[x_{k}^{\prime} \varphi_{i}^{\prime}\left(y_{k}\right) \Psi^{\prime}\left(u_{k}\right) \varphi_{j}^{\prime}\left(y_{k+1}\right)\right]^{\prime}$ and on the left by its transpose, with $i=\sigma(k)$ and $j=\sigma(k+1)$, it leads to

$$
\delta_{k} V-2 \mathrm{SC}_{u_{k}}-2 \mathrm{SC}\left(\varphi_{\sigma(k+1)}(\cdot), y_{k+1}, W_{\sigma(k+1)}\right)-2 \mathrm{SC}\left(\varphi_{\sigma(k)}(\cdot), y_{k}, T_{\sigma(k)}\right) \leq 0 .
$$

By applying a Schur complement on Inequality (17), one has

$$
\left[\begin{array}{cc}
P_{i} & C_{i}^{\prime} \Omega_{i}\left[\Delta_{i}-Q_{i}\right] \\
\star & 2 Q_{i}
\end{array}\right]-\frac{1}{\rho_{(\ell)}^{2}}\left(\widehat{K}_{i}-\widehat{J}_{i}\right)_{(\ell)}^{\prime}\left(\widehat{K}_{i}-\widehat{J}_{i}\right)_{(\ell)}>0_{(n+p)},
$$

with $\widehat{K}_{i}$ and $\widehat{J}_{i}$ as defined in Lemma 2.5. By multiplying on the right by $\widehat{x}_{k}=\left[x_{k}^{\prime} \varphi_{i}^{\prime}\left(y_{k}\right)\right]^{\prime}$ and on the left by its transpose, with $i=\sigma(k)$, it leads to

$$
V\left(\sigma(k) ; x_{k} ; \varphi_{\sigma(k)}\left(y_{k}\right)\right)+2 \operatorname{SC}\left(\varphi_{\sigma(k)}(\cdot), y_{k}, Q_{\sigma(k)}\right) \geq \frac{1}{\rho_{(\ell)}^{2}}\left\|\left(\widehat{K}_{\sigma(k)}-\widehat{J}_{\sigma(k)}\right)_{(\ell)} \widehat{x}_{k}\right\|^{2} .
$$

Because the $\varphi_{\sigma(k)}(\cdot)$ verifies the sector condition, one has

$$
V\left(\sigma(k) ; x_{k} ; \varphi_{\sigma(k)}\left(y_{k}\right)\right) \geq \frac{1}{\rho_{(\ell)}^{2}}\left\|\left(\widehat{K}_{\sigma(k)}-\widehat{J}_{\sigma(k)}\right)_{(\ell)} \widehat{x}_{k}\right\|^{2}
$$

which induces the inclusion

$$
L_{V}(1) \subset \mathcal{S}\left(\left\{\left(\widehat{K}_{\sigma}-\widehat{J}_{\sigma}\right)\right\}_{\sigma \in \mathcal{I}_{N}}, \rho\right)
$$

that is, in the set $L_{V}(1)$, the dead-zone sector condition (8) is verified.

By multiplying Inequality (16) on the right by $\widehat{x}_{0}=\left[x_{0}^{\prime} \varphi_{i}^{\prime}\left(C_{i} x_{0}\right)\right]^{\prime}$ and on the left by its transpose, with $i=\sigma(0)$, one has

$$
\mu x_{0}^{\prime} x_{0}+2 \operatorname{SC}\left(\varphi_{\sigma(0)}(\cdot), C_{\sigma(0)} x_{0}, R_{\sigma(0)}\right) \geq V\left(\sigma ; x_{0} ; \varphi_{\sigma(0)}\left(C_{\sigma(0)} x_{0}\right)\right) .
$$


The nonlinearity $\varphi_{\sigma(0)}(\cdot)$ verifying the sector condition $(3), \forall \sigma \in \mathcal{I}_{N}$, we have

$$
\mathcal{E}\left(\mu I_{n}\right) \subset L_{V}(1)
$$

By having the sector condition $\mathrm{SC}_{u_{k}} \leq 0$ verified inside $L_{V}(1)$, Inequality (21) implies $\delta_{k} V<0$ $(\forall x \neq 0)$, proving asymptotic stability in the set $L_{V}(1)$. Finally, by minimizing $\mu$, one has the maximization of the radius of the ball $\mathcal{E}\left(\mu I_{n}\right)$ included in $L_{V}(1)$.

Remark 2: Due to the fact that the control gains $K_{i}, \Gamma_{i}$ and the weighting matrices $U_{i}$ in $S C_{u_{k}}$ are fixed, $\forall i \in \mathcal{I}_{N}$, the Inequality (18) is a LMI, $\forall(i, j) \in \mathcal{I}_{N}^{2}$. Matrices $U_{i}$ can be set to the values obtained by the control design algorithm, like in (Jungers et al. 2009). The optimization problem of Theorem 3.1 can be considered to solve Problem 2.7, though the control gains must be also determined and (18) becomes a BMI, which means loss of convexity.

An example is presented to show that Theorem 3.1 is able to estimate the basin of attraction, for a fixed control law, solving the Problem 2.6. The control gains are the ones associated with the optimal solution of the framework proposed in (Jungers et al. 2009).

Example 1: Consider a switching system with $N=n=2 ; p=m=1 ; \rho=1.5, \Omega_{1}=0.7$; $\Omega_{2}=1.3 ; C_{1}=[0.90 .5] ; C_{2}=[1-0.7]$;

$$
\begin{aligned}
& A_{1}=\left[\begin{array}{cc}
0.4 & 0.4 \\
0.2 & 1
\end{array}\right] ; A_{2}=\left[\begin{array}{ll}
1.1 & 0.6 \\
0.3 & 0.4
\end{array}\right] ; B_{1}=\left[\begin{array}{l}
0.5 \\
0.5
\end{array}\right] ; \\
& B_{2}=\left[\begin{array}{l}
0.7 \\
0.5
\end{array}\right] ; F_{1}=\left[\begin{array}{c}
1 \\
1.2
\end{array}\right] ; F_{2}=\left[\begin{array}{c}
1.2 \\
1
\end{array}\right] .
\end{aligned}
$$

The modal nonlinearities are $\varphi_{1}(y)=0.5 \Omega_{1} y\left(1-\exp \left(-0.2 y^{2}\right) ; \varphi_{2}(y)=0.5 \Omega_{2} y\left(1+\cos \left(\frac{100 y}{3}\right)\right)\right.$. The control synthesis algorithm in (Jungers et al. 2009), based on a switched quadratic Lyapunov function, provides $\mu=2.7140$ with the control gains $\Gamma_{1}=-1.1411 ; K_{1}=[-0.9266-0.9892]$; $K_{2}=[-1.7245-0.5038] ; \Gamma_{2}=-1.1890$. We wish to estimate the basin of attraction of this system. Hence, by applying Theorem 3.1 , with matrices $U_{i} \in \mathbb{R}^{m \times m}$ set to the values given by in (Jungers et al. 2009), $\forall i \in \mathcal{I}_{N}$, one gets $\mu=2.3789$.

Both estimates are shown in Fig. 1. The largest ball $\mathcal{E}\left(\mu I_{n}\right)$ solution of our optimization problem is also depicted (dashed line). It is possible to see that, for this example, the intersection of ellipsoids given by (Jungers et al. 2009) (dot-dashed line) is included in the $L_{V}(1)$ (solid line). By calculating the area of both regions $\mathcal{A}_{L_{V}(1)}=2.6135$ and $\mathcal{A}_{\mathcal{E}}=1.8957$ of (Jungers et al. 2009). One can conclude that the area has been increased by $37.87 \%$.

\section{Closed-loop Stabilization}

This section is devoted to tackle Problem 2.7 based on the function (9). According to Remark 2, this task is naturally described as BMIs, which could be then sub-optimally solved by considering an iterative algorithm (Tarbouriech et al. 2011). However, it is known that a solution provided by this approach is highly sensitive to the initial values of the algorithm. Hence, a relaxation is proposed by means of the Finsler's Lemma (de Oliveira and Skelton 2001) leading to sufficient conditions given by LMIs which are considered in the following statement.

Theorem 4.1 If there exists, $\forall i \in \mathcal{I}_{N}$, matrices $\mathcal{F}_{G, i}, X_{G, i} \in \mathbb{R}^{n \times n}, K_{i}, J_{1, i} \in \mathbb{R}^{m \times n}, \Gamma_{i}, J_{2, i} \in$ $\mathbb{R}^{m \times p}$, symmetric positive definite matrices $P_{i} \in \mathbb{R}^{n \times n}$ and positive semi-definite diagonal matrices $\Delta_{i} \in \mathbb{R}^{p \times p}$ and positive definite diagonal matrices $X_{\Theta, i}, \mathcal{F}_{\Theta, i}, R_{i}, Q_{i}, T_{i} \in \mathbb{R}^{p \times p}, U_{i} \in \mathbb{R}^{m \times m}$ and a scalar $\mu$ solutions of the convex optimization problem

$$
\min _{\mu, \mathcal{F}_{G, i}, X_{G, i}, P_{i}, K_{i}, J_{1, i}, \Gamma_{i}, J_{2, i}, Q_{i}, R_{i}, T_{i}, U_{i}, \mathcal{F}_{\Theta, i}, X_{\Theta, i}, \Delta_{i}} \mu
$$




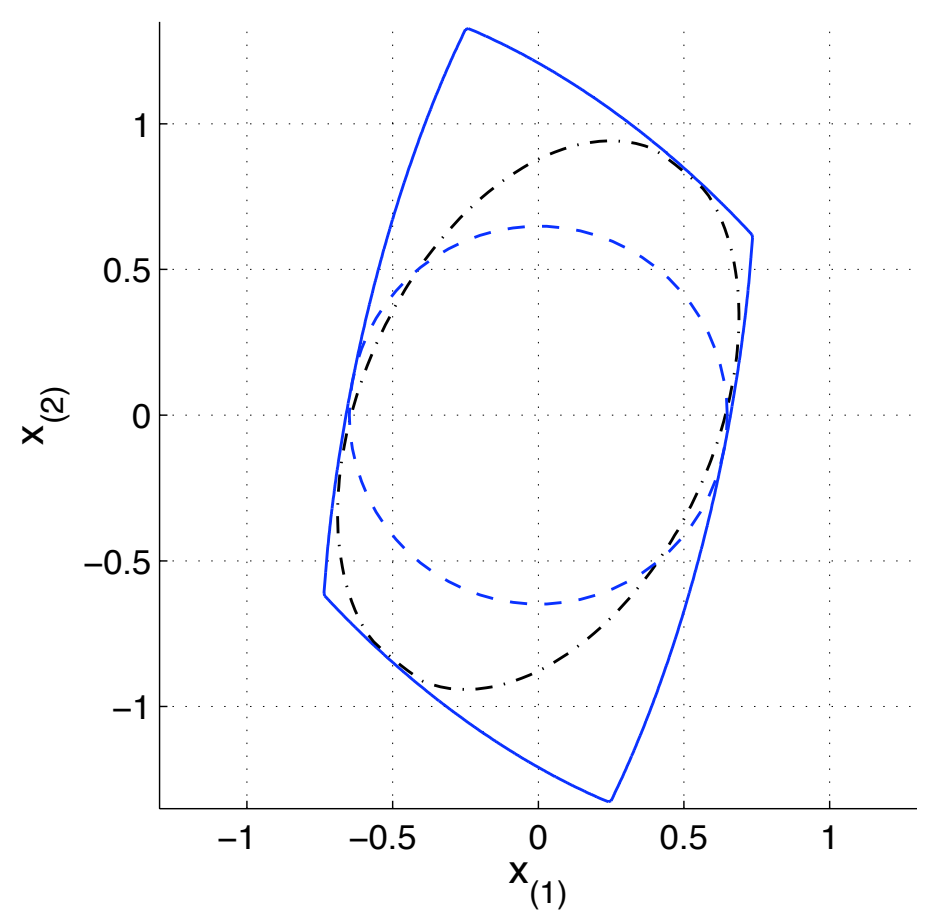

Figure 1. Level set $L_{V}(1)$ of Theorem 4.1 (solid line). Ellipsoids intersection of (Jungers et al. 2009) (dot-dashed line) and largest sphere $\mathcal{E}\left(\mu I_{n}\right) \subset L_{V}(1)$ given by Theorem 4.1 (dashed line).

subject to LMIs: (16), (17), and

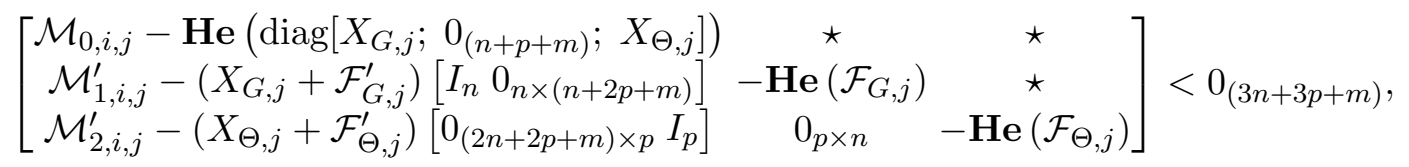

$\forall(i, j) \in \mathcal{I}_{N}^{2}$. Then, with

$$
G_{i}=X_{G, i} \mathcal{F}_{G, i}^{-1} ; \quad \Theta_{i}=X_{\Theta, i} \mathcal{F}_{\Theta, i}^{-1}, \forall i \in \mathcal{I}_{N}
$$

Problem 2.7 is fulfilled with an estimate of $\mathcal{B}_{0}$ given by the set $L_{V}(1)$.

Proof The Inequalities (16)-(17) remain as stated in Theorem 3.1, implying the Inclusions (25) and (27). Moreover, if Inequality (28) holds, it means that there exists a multiplier

$$
\mathcal{F}_{i, j}=\left[\begin{array}{cc}
\mathcal{F}_{G, j} & 0_{(2 n+p+m) \times p} \\
0_{(n+2 p+m) \times n} & \mathcal{F}_{\Theta, j} \\
\mathcal{F}_{G, j} & 0_{n \times p} \\
0_{p \times n} & \mathcal{F}_{\Theta, j}
\end{array}\right],
$$

with $\mathcal{F}_{G, j} \in \mathbb{R}^{n \times n}$ of full rank and $\mathcal{F}_{\Theta, j} \in \mathbb{R}^{p \times p}$ diagonal positive definite, verifying

$$
\left[\begin{array}{ccc}
\mathcal{M}_{0, i, j} & \mathcal{M}_{1, i, j} & \mathcal{M}_{2, i, j} \\
\mathcal{M}_{1, i, j}^{\prime} & 0_{n} & 0_{n \times p} \\
\mathcal{M}_{2, i, j}^{\prime} & 0_{p \times n} & 0_{p}
\end{array}\right]+\mathbf{H e}\left(\mathcal{F}_{i, j}\left[\begin{array}{ccc}
\mathcal{N}_{G_{j}} & -I_{n} & 0_{n \times p} \\
\mathcal{N}_{\Theta_{j}} & 0_{p \times n} & -I_{p}
\end{array}\right]\right)<0_{(3 n+3 p+m)},
$$


where $\mathcal{N}_{G_{j}}$ and $\mathcal{N}_{\Theta_{i}}$ are as previously defined. By the Finsler's Lemma, this is equivalent to have

$$
\left[\begin{array}{c}
I_{2 n+2 p+m} \\
\mathcal{N}_{G_{j}} \\
\mathcal{N}_{\Theta_{j}}
\end{array}\right]^{\prime}\left[\begin{array}{ccc}
\mathcal{M}_{0, i, j} & \mathcal{M}_{1, i, j} & \mathcal{M}_{2, i, j} \\
\mathcal{M}_{1, i, j}^{\prime} & 0_{n} & 0_{n \times p} \\
\mathcal{M}_{2, i, j}^{\prime} & 0_{p \times n} & 0_{p}
\end{array}\right]\left[\begin{array}{c}
I_{2 n+2 p+m} \\
\mathcal{N}_{G_{j}} \\
\mathcal{N}_{\Theta_{j}}
\end{array}\right]<0_{(2 n+2 p+m)}
$$

verified, and which is, in fact, the Inequality (18). Hence, one can conclude that Theorem 4.1 allows to exhibit a solution for Problem 2.7 by considering the proof of Theorem 3.1.

Let us reconsider the Example 1 to perform the control synthesis by applying Theorem 4.1. It will illustrate some special features of this novel Lyapunov function.

Control synthesis for Example 1: The optimization problem of Theorem 4.1 provides $\mu=2.5563$ with the control gains given by $K_{1}=[-0.7168-1.0136] ; \Gamma_{1}=-1.2923$; $K_{2}=[-1.2581-0.7326] ; \Gamma_{2}=-1.4650$. The estimate $L_{V}(1)$ is depicted (solid line) in Fig. 2 with largest sphere $\mathcal{E}\left(\mu I_{n}\right) \subset L_{V}(1)$ (dashed line) and the estimate set given by Theorem 3.1 (dotted line).

It is possible to see that our estimate presents disconnected sets and also contains the ellipsoids intersection of (Jungers et al. 2009) (dot-dashed line). By calculating the area, one has $\mathcal{A}_{L_{V}(1)}=$ 3.2471 , corresponding an improvement of $61 \%$ of with respect to the one of (Jungers et al. 2009) and $24 \%$ regarding the area related to the estimate of Theorem 3.1 given in Section 3.

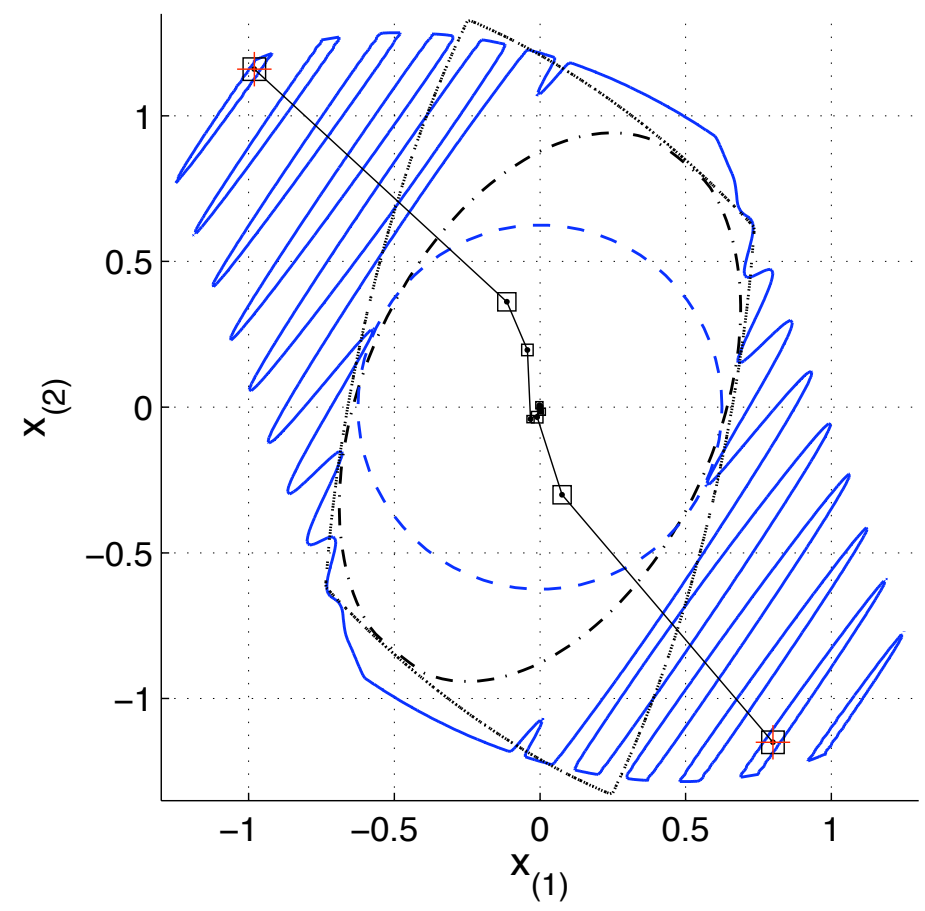

Figure 2. Set $L_{V}(1)$ obtained by Theorem 4.1 (solid line) and Theorem 3.1 (dotted line). Ellipsoids intersection of (Jungers et al. 2009) (dot-dashed line). Two stable trajectories under two realizations of arbitrary switching rules.

Two trajectories of the closed-loop system (6) with the control gains calculated by our framework and two different realizations of arbitrary switching rules are also depicted in Fig. 2. The initial conditions are settled in different disconnected sets of our estimate. It is noteworthy that, the trajectories converge asymptotically to the origin.

Because Theorem 4.1 provides sufficient stability conditions, a question about the gap between the basin of attraction $\mathcal{B}_{0}$ and the disconnected estimate set $L_{V}(1)$ may arise. Hence, we have analysed the trajectories for initial conditions located in the following region in the phase portrait: $-2 \leq x_{0,(1)} \leq 2$ and $-2 \leq x_{0,(2)} \leq 2$ (including the set $L_{V}(1)$ ). Four switching rules were considered: 
- $\sigma_{a}: \sigma_{a}(k)=1 ; \forall k \in \mathbb{N}$;

- $\sigma_{b}: \sigma_{b}(k)=2 ; \forall k \in \mathbb{N}$;

- $\sigma_{c}: \sigma_{c}(2 k)=1 ; \sigma_{c}(2 k+1)=2 ; \forall k \in \mathbb{N}$;

- $\sigma_{d}: \sigma_{d}(2 k)=2 ; \sigma_{d}(2 k+1)=1 ; \forall k \in \mathbb{N}$.

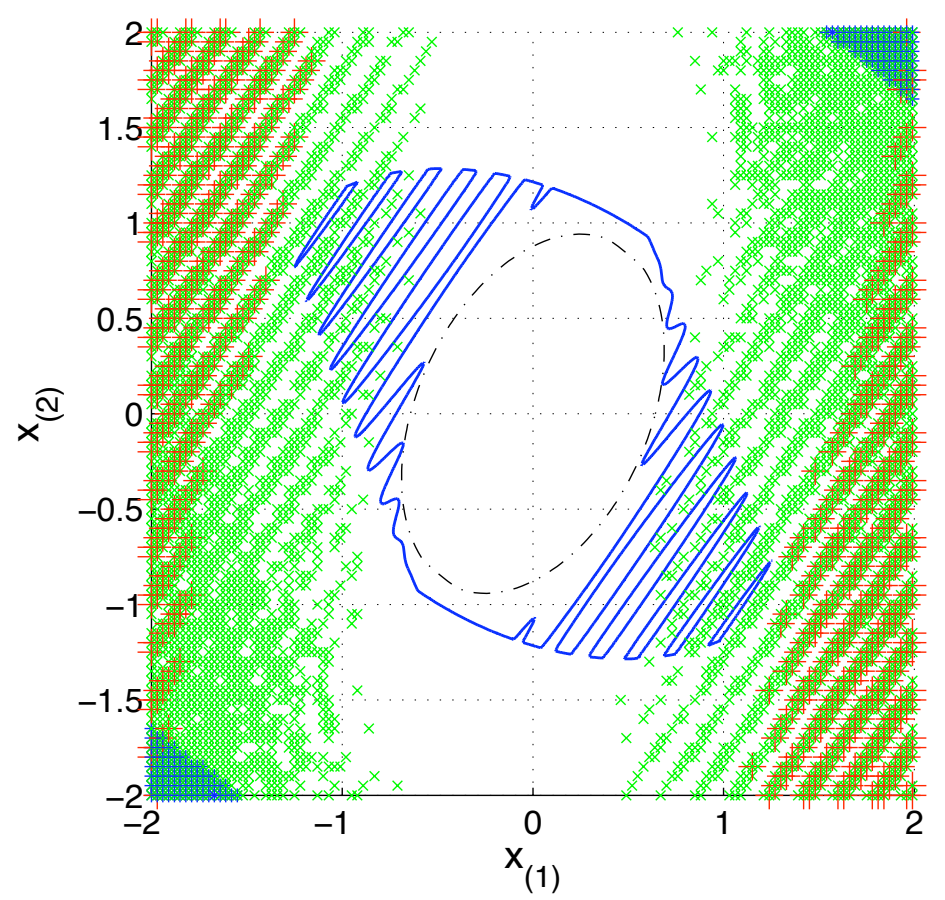

Figure 3. Set $L_{V}(1)$ (solid line) and initial conditions $x_{0}=\left[x_{0,(1)} ; x_{0,(2)}\right]^{\prime}$ leading to unstable trajectories. For the switching rules $\sigma_{a}$ (in blue), $\sigma_{b}$ (in green), $\sigma_{c}$ and $\sigma_{d}$ (in red).

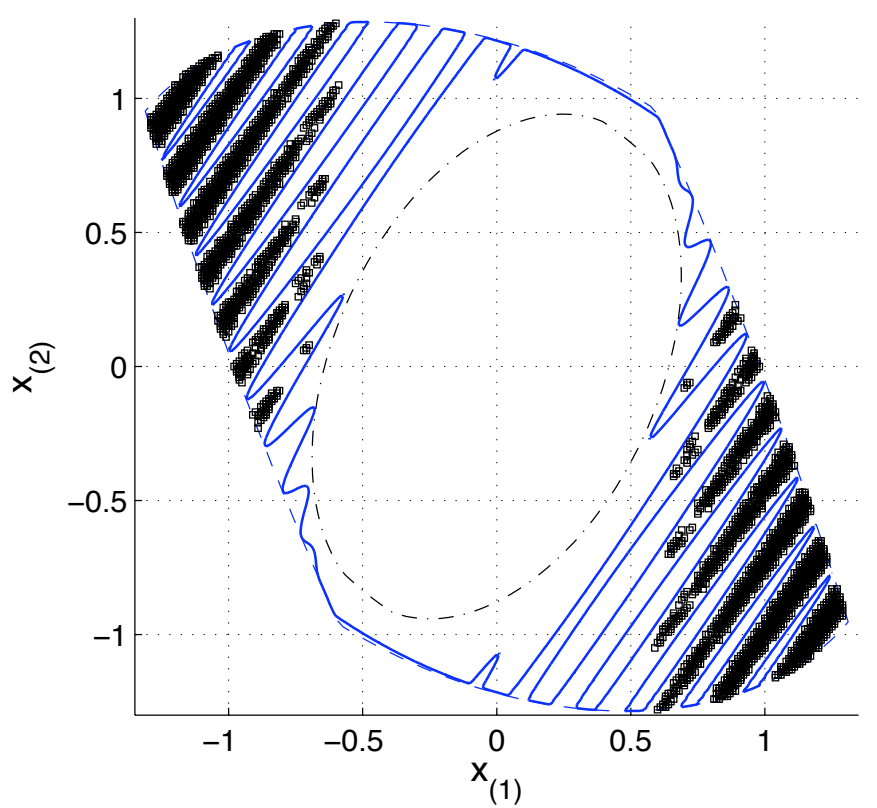

Figure 4. Zoom of Fig. 3: Set $L_{V}(1)$ (solid line) obtained by Theorem 4.1. Ellipsoids intersection of (Jungers et al. 2009) (dot-dashed line). Unstable trajectories between the disconnected $L_{V}(1)$.

It can be seen in Fig. 3 numerous points (shadow region) $x_{0} \notin L_{V}(1)$ for which system (6) 
is unstable under one of the considered switching rules. In fact, these several points filling up the region between the disconnected sets, in Fig. 4, point out the suitability of the set $L_{V}(1)$ in estimating the basin of attraction. Thus, one has highlighted a remarkable characteristic of our Lyapunov function which is a less conservative estimate of $\mathcal{B}_{0}$ of the system (6).

\section{Conclusion}

The class of discrete-time switched systems with mode-dependent cone bounded nonlinearities subject to input saturation was addressed in this paper. As main contribution, a new switched Lyapunov function is considered in order to take the switched nonlinearities into account. The great feature of this function, in respect to the Lur'e-type one, is the possibility of covering modedependent nonlinearities and requiring only the mode-dependent sector condition assumption. Local stability analysis and control design were tackled by optimization problems aiming to enlarge the size of the basin of attraction estimate. This estimate is induced by the Lyapunov function level set and it may be composed of disconnected sets. Stability analysis is given as LMIs conditions which were used in convex programming. For the stabilization purposes, the Finsler's Lemma was applied with a particular structure of multipliers such that sufficient LMI conditions were achieved. Numerical examples illustrate the relevance of our Lyapunov function regarding the switched quadratic function.

\section{Acknowledgement}

This work was partially supported by ANR project ArHyCo, Programme "Systèmes Embarqués et Grandes Infrastructures" - ARPEGE, contract number ANR-2008 SEGI 004 01-30011459 and by the European Community's Seventh Framework Programme (FP7/2007-2013) under grant agreement $n^{\circ}$ 257462: HYCON2 Network of Excellence "Highly-Complex and Networked Control Systems".

\section{References}

Arcak, M., Larsen, M., and Kokotovic, P. (2003), "Circle and Popov criteria as tools for nonlinear feedback designs," Automatica, 39, 643-650.

Benzaouia, A., Saydy, L., and Akhrif, O. (2004), "Stability and control synthesis of switched systems subject to actuator saturation," in American Control Conference, June, Boston, Massachusetts, USA, pp. 5818-5823.

Boyd, S., El Ghaoui, L., Feron, E., and Balakrishnan, V., Linear Matrix Inequalities in System and Control Theory, SIAM Studies in Applied Mathematics (1994).

Daafouz, J., and Bernussou, J. (2001), "Parameter dependent Lyapunov functions for discrete time systems with time varying parametric uncertainties," Systems $\&$ Control Letters, 43, 355-359.

de Oliveira, M.C., and Skelton, R. (2001), "Stability tests for constrained linear systems," in Perspectives in Robust Control, ed. S.O.R. Moheimani, Lectures Notes in Control an Information Sciences, Springer-Verlag, pp. 241-257.

Gomes da Silva Jr., J.M., Paim, C., and Castelan, E.B. (2001), "Stability and Stabilization of Linear Discrete-Time subject to Control Saturation," in 1st IFAC Symp. on System Structure and Control (SSSC'01), Prague.

Gonzaga, C.A.C., Jungers, M., and Daafouz, J. (2011), "Stability analysis of discrete-time Lur'e systems," In revision for Automatica.

Haddad, W.M., and Bernstein, D.S. (1994), "Parameter-dependent Lyapunov Functions and the Discrete-time Popov Criterion for Robust Analysis," Automatica, 30, 1015-1021. 
Jungers, M., Castelan, E.B., Tarbouriech, S., and Daafouz, J. (2009), "Stabilization of discretetime swithing systems including modal nonlinearities and saturation actuators," in 3rd IFAC Conference on Analysis and Design of Hybrid Systems, Zaragoza, Spain, pp. 174-179.

Jungers, M., Castelan, E.B., Tarbouriech, S., and Daafouz, J. (2010), "Finite $\mathcal{L}_{2}$-induced gain and $\lambda$-contractivity of discrete-time switching systems including modal nonlinearities and actuator saturations," Nonlinear Analysis: Hybrid Systems, 5, 289-300.

Jury, E.I., and Lee, B.W. (1964a), "On the absolute stability of a certain class of nonlinear sampled-data systems.," IEEE Transactions on Automatic Control, 9, 51-61.

Jury, E.I., and Lee, B.W. (1964b), "On the absolute stability of nonlinear sampled-data systems.," IEEE Transactions on Automatic Control, pp. 551-554.

Kapila, V., and Haddad, W.M. (1996), "A Multivariable Extension of the Tsypkin Criterion Using a Lyapunov-Function Approach," IEEE Transactions on Automatic Control, 41, 149152.

Khalil, H.K., Nonlinear Systems - Third Edition, Prentice Hall (2002).

Liberzon, D., Switching in Systems and Control, Vol. in series Systems and Control: Foundations and Applications., Birkhäuser, Boston, MA (2003).

Lu, L., and Lin, Z. (2008), "Design of switched linear systems in the presence of actuator saturation," IEEE Transactions on Automatic Control, 53, 1536-1542.

Sharma, T.N., and Singh, V. (1981), "On the absolute stability of multivariable discrete-time nonlinear systems.," IEEE Transactions on Automatic Control, AC-26, 51-61.

Tarbouriech, S., Garcia, G., Gomes da Silva Jr, J.M., and Queinnec, I., Stability and Stabilization of Linear Systems with Saturating Actuators, 1st edition ed., Springer Verlag (2011).

Tarbouriech, S., Prieur, C., and Gomes da Silva Jr., J.M. (2006), "Stability analysis and stabilization of systems presenting nested saturations," IEEE Transactions on Automatic Control, $51,1364-1371$. 